\title{
Effect of dissipation factor on reflected and transmitted powers of a structure containing left-handed material waveguide
}

\author{
Muin F. Ubeid ${ }^{1, *}$, Mohammed M. Shabat ${ }^{1}$, Mohammed O. Sid-Ahmed ${ }^{2}$ \\ ${ }^{1}$ Department of Physics, Faculty of Science, Islamic University of Gaza, P.O. 108, Gaza, \\ Fax: + 790-082823311, Gaza Strip, Palestinian Authority \\ ${ }^{2}$ Department of Physics, Faculty of Science, Sudan University of Science and Technology, \\ Republic of Sudan \\ *E-mail address: mubeid@mail.iugaza.edu
}

\begin{abstract}
In this paper a waveguide structure consisting of a pair of left-handed material (LHM) and dielectric slabs inserted in vacuum is investigated theoretically. Two cases of the LHM are considered, loss-less case and loss case as well as the frequency dependence of permittivity and permeability of it is taken into account. Maxwell's equations are used to determine the electric and magnetic fields of the incident waves at each layer. Snell's law is applied and the boundary conditions are imposed at each layer interface to calculate the reflected, transmitted and loss powers of the structure. Numerical results are illustrated to show the effect of frequency, angle of incidence and LHM thickness on the mentioned powers when the dissipation factor changes. The obtained results are in agreement with the law of conservation of energy.
\end{abstract}

Keywords: Electromagnetic waves; left-handed material; dissipation factor; frequency; Reflected power; transmitted power

\section{INTRODUCTION}

Metamaterials (sometimes termed left-handed materials (LHMs)) are materials whose permittivity $\varepsilon$ and permeability $\mu$ are both negative and consequently have negative index of refraction. These materials are artificial and theoretically discussed first by Veselago [1] over 40 years ago. The first realization of such materials, consisting of split-ring resenators (SRRs) and continuous wires, was first introduced by Pendry $[2,3]$.

Regular materials are materials whose $\varepsilon$ and $\mu$ are both positive and termed right handed materials (RHMs). R. A. Shelby et al [4] have studied negative refraction in LHMs. I. V. Shadrivov [5] has investigated nonlinear guided waves in LHMs. N. Garcia et al [6] have shown that LHMs don't make a perfect lens.

Kong [7] has provided a general formulation for the electromagnetic wave interaction with stratified metamaterial structures. M. F. Ubeid et al [8] have discussed the propagation of electromagnetic waves through a dielectric counterpart of left-handed material. 
I. Kourakis et al [9] have investigated a nonlinear propagation of electromagnetic waves in negative-refraction index LHM. M. F. Ubeid et al [10] have demonstrated the effect of negative permittivity and permeability on the transmission of electromagnetic waves through a structure containing left-handed material.

H. Cory et al [11] and C. Sabah et al [12] have estimated high reflection coatings of multilayered structure. Oraizi et al [13] have obtained a zero reflection from multilayered metamaterial structures.

In this paper we consider a structure consisting of LHM and dielectric slabs inserted in vacuum. A plane polarized wave is obliquely incident on it. Tow cases of the LHM are considered, loss-less case and loss case.

Maxwell's equations are used to determine the electric and magnetic fields in each region. Then, Snell's law is applied and boundary conditions are imposed at each interface to obtain a number of equations with unknown parameters.

The equations are solved for the unknown parameters to calculate the reflection and transmission coefficients. These coefficients are used to determine the reflected, transmitted and loss powers of the structure.

The effect of many parameters like frequency, angle of incidence etc. on the mentioned powers are studied in details by changing the dissipation factor of the LHM.

Throughout the computations, the frequency dependence of permittivity and permeability of the LHM are taken into account. The numerical results are in agreement with the law of conservation of energy given by $[11,14,15]$. It is also noticed that the numerical results of Figure 4 is similar to Figure 8.6 obtained by [14], this is another evidence for validity of the performed computations.

The propagation of electromagnetic waves through a structure containing LHM and dielectric is considered by many previous studies.

Our work is concentrated on the powers of the structure and the role of properties of the dissipation factor of LHM layer.

Thus, the behavior of the powers for the incident waves against the incident angle, the frequency and LHM thickness is computed and presented in numerical results with the emphasis on the dissipation factor of the LHM.

\section{THEORY}

We consider four regions each with permittivity $\varepsilon_{\ell}$ and permeability $\mu_{\ell}$, where $\ell$ represents the region order. Region 1 and 4 are vacuums $\left(\varepsilon_{o}, \mu_{o}\right)$, Region 2 is a regular dielectric $\left(\varepsilon_{2}, \mu_{2}\right)$, Region 3 is a metamaterial $\left(\varepsilon_{3}(\omega), \mu_{3}(\omega)\right)$.

A polarized plane wave in Region 1 incident on the plane $\mathrm{z}=0$ at some angle $\theta$ relative to the normal to the boundary (see Fig. 1). 


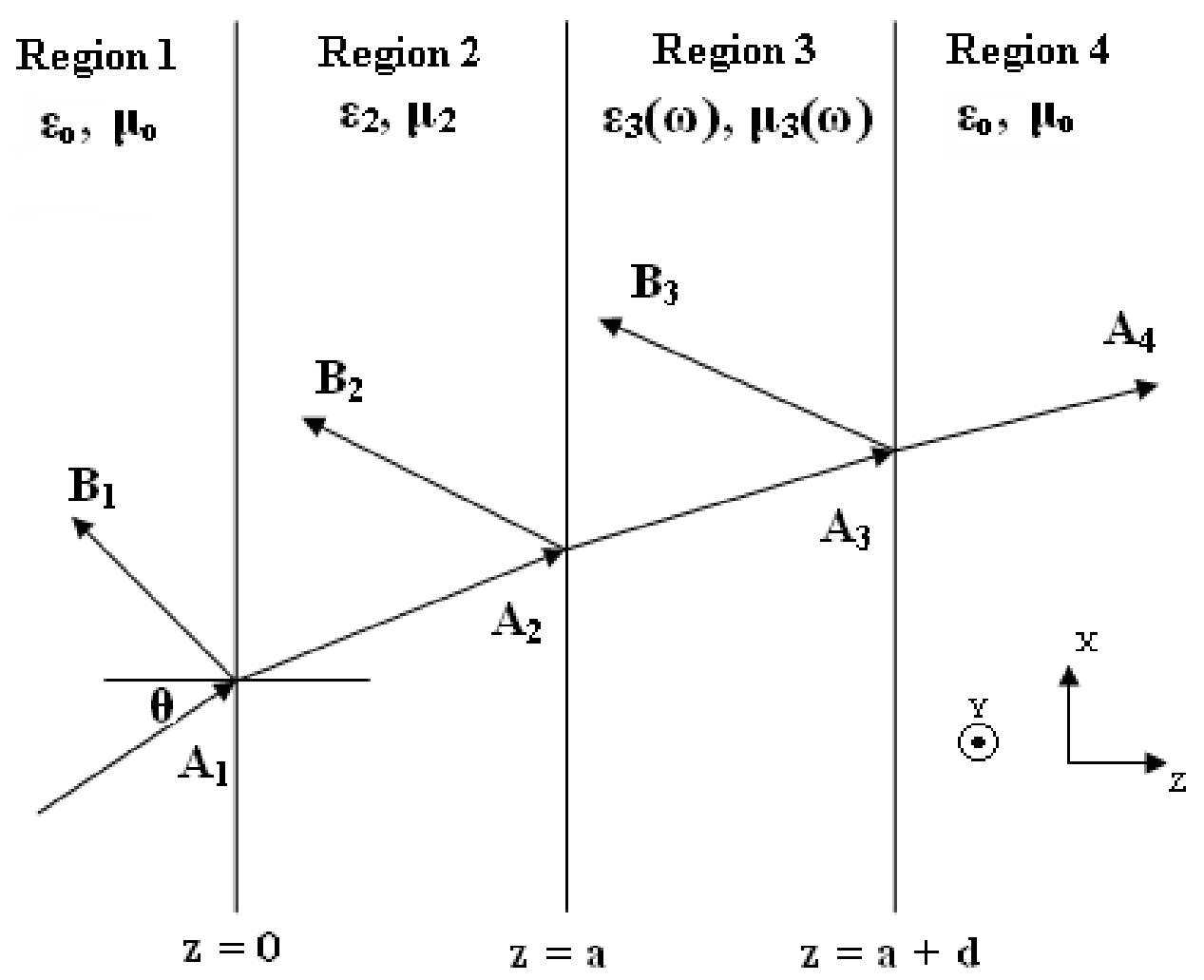

Fig. 1. Wave propagation through a structure consisting of a pair of dielectric and metamaterial embedded in vacuum.

The electric field in each region is [7, 11]:

$$
\vec{E}_{\ell}=\left(A_{\ell} e^{i k_{\ell z} z}+B_{\ell} e^{-i k_{\ell z} z}\right) e^{i\left(k_{\ell x} x-\omega t\right)} \hat{y}
$$

We use Maxwell's equation as done by [16] to find corresponding magnetic field $\vec{H}_{\ell}$ :

$$
\vec{H}_{\ell}=\frac{1}{\mu_{\ell} \omega}\left[\left(A_{\ell} k_{\ell x} e^{i k_{\ell z} z}+B_{\ell} k_{\ell x} e^{-i k_{\ell z} z}\right) \hat{z}+\left(-A_{\ell} k_{\ell z} e^{i k_{\ell z} z}+B_{\ell} k_{\ell z} e^{-i k_{\ell z} z}\right) \hat{x}\right] e^{i\left(k_{\ell x} x-\omega t\right)}
$$

Where $A_{\ell}$ and $B_{\ell}$ are the amplitudes of forward and backward traveling waves. $k_{\ell}=n_{\ell} \omega / c$ is the wave vector inside the material and $n_{\ell}$ is the refractive index of it. Matching the boundary conditions for $\vec{E}$ and $\vec{H}$ fields at each layer interface, that is at $\mathrm{z}=0, E_{1}=E_{2}$ and $H_{1}=H_{2}$ and so on. This yields six equations with six unknown parameters $[11,13,16]$ :

$$
A_{1}+B_{1}=A_{2}+B_{2}
$$




$$
\begin{gathered}
\frac{k_{1 z}}{\mu_{1}}\left(A_{1}-B_{1}\right)=\frac{k_{2 z}}{\mu_{2}}\left(A_{2}-B_{2}\right) \\
A_{2} e^{i k_{2 z} d_{2}}+B_{2} e^{-i k_{2 z} d_{2}}=A_{3} e^{i k_{3 z} d_{2}}+B_{3} e^{-i k_{3 z} d_{2}} \\
\frac{k_{2 z}}{\mu_{2}}\left(A_{2} e^{i k_{2 z} d_{2}}-B_{2} e^{-i k_{2 z} d_{2}}\right)=\frac{k_{3 z}}{\mu_{3}}\left(A_{3} e^{i k_{3 z} d_{2}}-B_{3} e^{-i k_{3 z} d_{2}}\right) \\
A_{3} e^{i k_{3 z}\left(d_{2}+d_{3}\right)}+B_{3} e^{-i k_{3 z}\left(d_{2}+d_{3}\right)}=A_{4} e^{i k_{4 z}\left(d_{2}+d_{3}\right)} \\
\frac{k_{3 z}}{\mu_{3}}\left(A_{3} e^{i k_{3 z}\left(d_{2}+d_{3}\right)}-B_{3} e^{-i k_{3 z}\left(d_{2}+d_{3}\right)}\right)=\frac{k_{4 z}}{\mu_{4}} A_{4} e^{i k_{4 z}\left(d_{2}+d_{3}\right)}
\end{gathered}
$$

Where $k_{1 x}=k_{2 x}=k_{3 x}=k_{4 x} \equiv$ Snell's law.

Letting $A_{1}=1$ and solving the obtained equations for the unknown parameters enables us to calculate the reflection and transmission coefficients $B_{1}$ and $A_{4}[11,16]$. The reflected power $\mathrm{R}$ and the transmitted power $\mathrm{T}$ are given by $[11,16]$ :

$$
R=B_{1} B_{1}^{*}, T=\left(k_{4 z} / k_{1 z}\right) A_{4} A_{4}^{*}
$$

Where $B_{1}{ }^{*}$ and $A_{4}{ }^{*}$ are the complex conjugate of $B_{1}$ and $A_{4}$ respectively.

The law of conservation of energy is given by $[11,14,15]$ :

$$
\mathrm{R}+\mathrm{T}=1-\mathrm{P}_{\text {loss }}
$$

Where, $\mathrm{P}_{\text {loss }}$ is the loss power due to losses in LHM and $k_{\ell z}$ is given by:

$$
k_{\ell z}=\frac{\omega}{c} \sqrt{n_{\ell}^{2}-n_{1}^{2} \sin ^{2} \theta_{1}}
$$

\section{NUMERICAL RESULTS}

For the LHM in region 3 we employ a dispersive one with $\varepsilon_{3}$ and $\mu_{3}$ appeared in $[2,3,16]$ :

$$
\varepsilon_{3}(\omega)=1-\frac{F_{e} \omega_{e p}^{2}}{\omega^{2}-\omega_{e o}^{2}+i \gamma_{e} \omega}
$$




$$
\mu_{3}(\omega)=1-\frac{F_{m} \omega_{m p}^{2}}{\omega^{2}-\omega_{m o}^{2}+i \gamma_{m} \omega}
$$

where $\omega_{e p}$ and $\omega_{m p}$ are the electric and magnetic plasma frequencies, $\omega_{e o}$ and $\omega_{m o}$ are the electric and magnetic resonance frequencies. $F_{e}$ and $F_{m}$ are the scaling filling parameters. $\gamma_{\mathrm{e}}$ and $\gamma_{\mathrm{m}}$ are the electric and magnetic dissipation factors.

We have used the following parameters appearing in [16]: $\omega_{m p}=2 \pi 10.95 \mathrm{GHz}, \omega_{m o}=$ $2 \pi 10.1 \mathrm{GHz}, F_{m}=.26, \omega_{e p}=2 \pi 13.3 \mathrm{GHz}, \omega_{e o}=2 \pi 10.3 \mathrm{GHz}, F_{e}=.37$. Two cases of the LHM are considered, loss-less case $\left(\gamma_{e}=\gamma_{m}=\gamma=0\right)$ and loss_case $\left(\gamma_{\mathrm{e}}=\gamma_{\mathrm{m}}=\gamma \neq 0\right)$. In the lossless case, the frequency range in which $\varepsilon_{3}(\omega)$ and $\mu_{3}(\omega)$ are negative extends from 10.3 up to $11.5 \mathrm{GHz}$.

The operating frequency is assumed to be $2 \pi 11 \mathrm{GHz}$. This frequency is chosen by an arbitrary decision, but it must be in the frequency band where the permittivity and permeability of the LHM are both simultaneously negative.

Region 1, 2 and 4 in Fig. 1 are assumed to be loss-less. The thickness of each of LHM and dielectric slabs is equal to one half-wavelength long at the operating frequency. $\mathrm{T}$ and $\mathrm{R}$ are calculated numerically as stated above.

In Fig. 2 we show a calculation of reflected, transmitted and loss powers of the considered structure as a function of frequency for three values of dissipation factor of the $\operatorname{LHM}(\gamma=0, .1$ and $.2 \mathrm{GHz})$ and for $30^{\circ}$ angle of incidence.

The frequency is changed between $9 \mathrm{GHz}$ and $12 \mathrm{GHz}$, because the simultaneously negative permittivity and permeability can be realized in this range in all cases, according to Eq (12) and (13). As confirmed from Fig. 2, the powers show increasing, decreasing and oscillatory behaviors in different frequency ranges.

The interpretation of this is as follows: the transmission is null above $11.5 \mathrm{GHz}$ frequency, where all incident radiation is being reflected. In this band $\varepsilon_{3}(\omega)$ and $\mu_{3}(\omega)$ are different in signs, and then $n_{3}$ is imaginary, this is because

$$
n_{3}=\sqrt{\varepsilon_{.3} \mu_{3} / \varepsilon_{0} \mu_{0}}
$$

In $10.3-11.5 \mathrm{GHz}$ band the transmission is very good. In this band $\varepsilon_{3}(\omega)$ and $\mu_{3}(\omega)$ are both negative, and then $n_{3}$ is real. At 10.1 and $10.2 \mathrm{GHz}$ frequencies the transmitted power is zero because $\mu_{3}(\omega)$ is positive and $\varepsilon_{3}(\omega)$ is negative and then $n_{3}$ is imaginary.

In 9-10 GHz band the transmitted power is not zero because both $\varepsilon_{3}(\omega)$ and $\mu_{3}(\omega)$ are positive and then $\mathrm{n}_{3}$ is real.

This means that electromagnetic waves will only propagate in a medium that has a real index of refraction [16]. This interpretation is presented for loss-less case and can be applied on the loss case of LHM. 


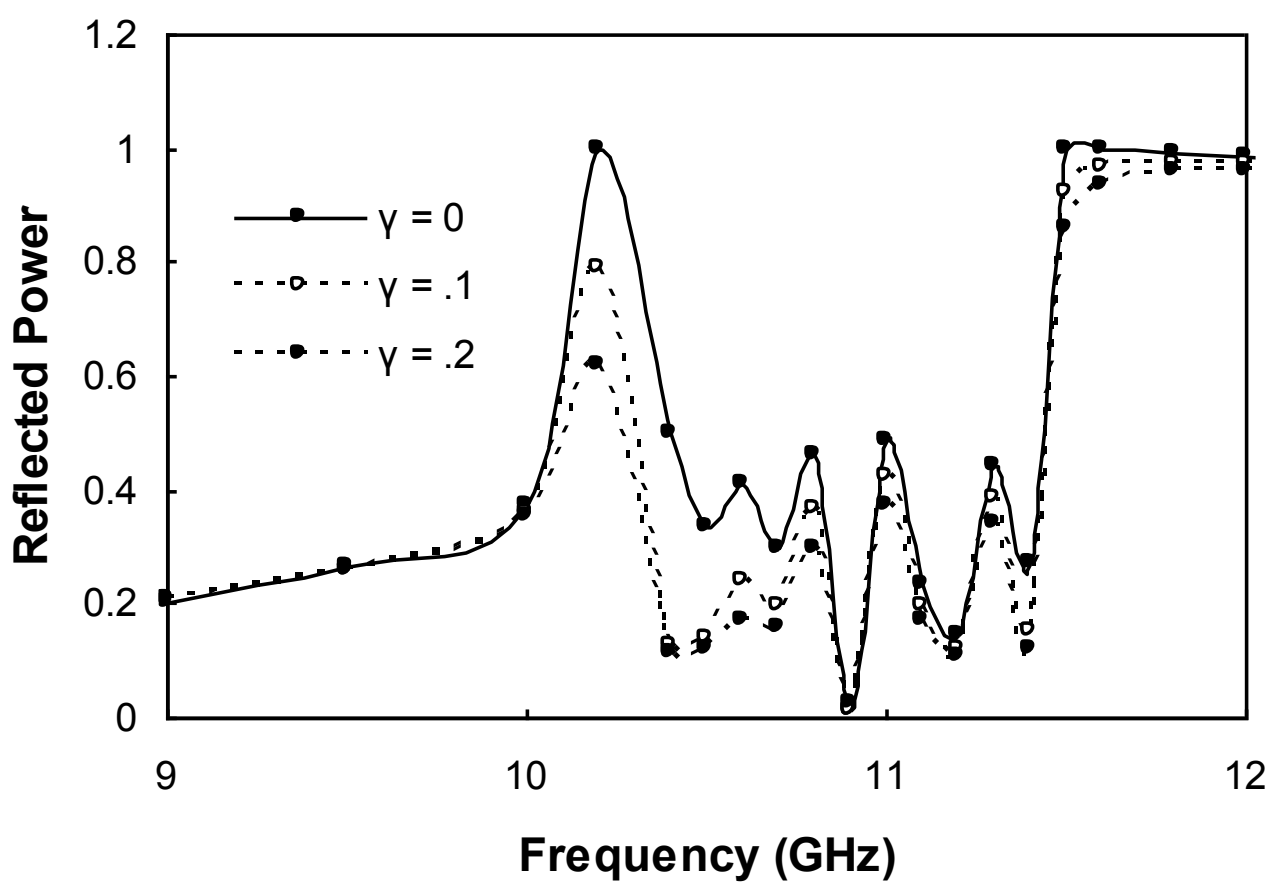

(a)

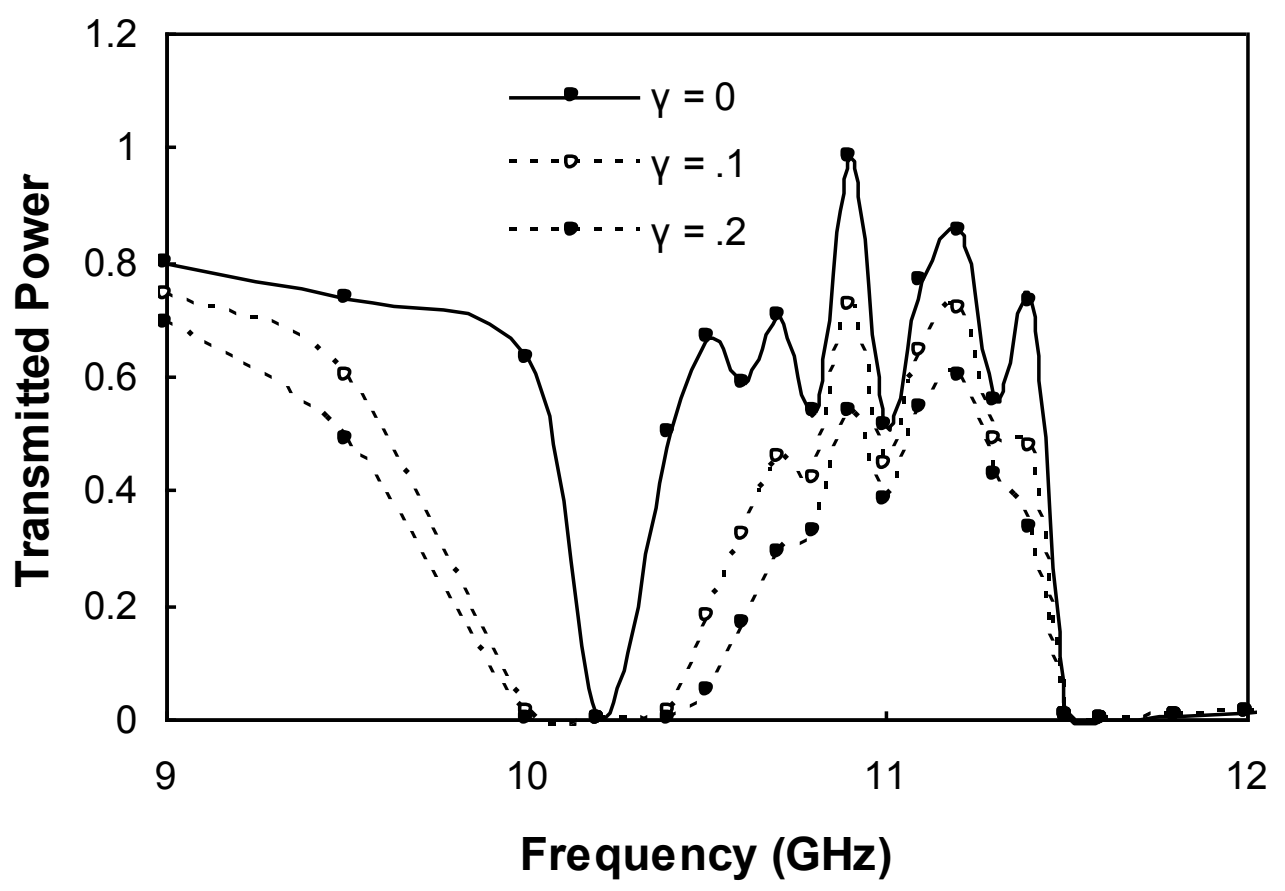

(b)

Fig. 2. Reflected, transmitted and loss powers as a function of frequency. 


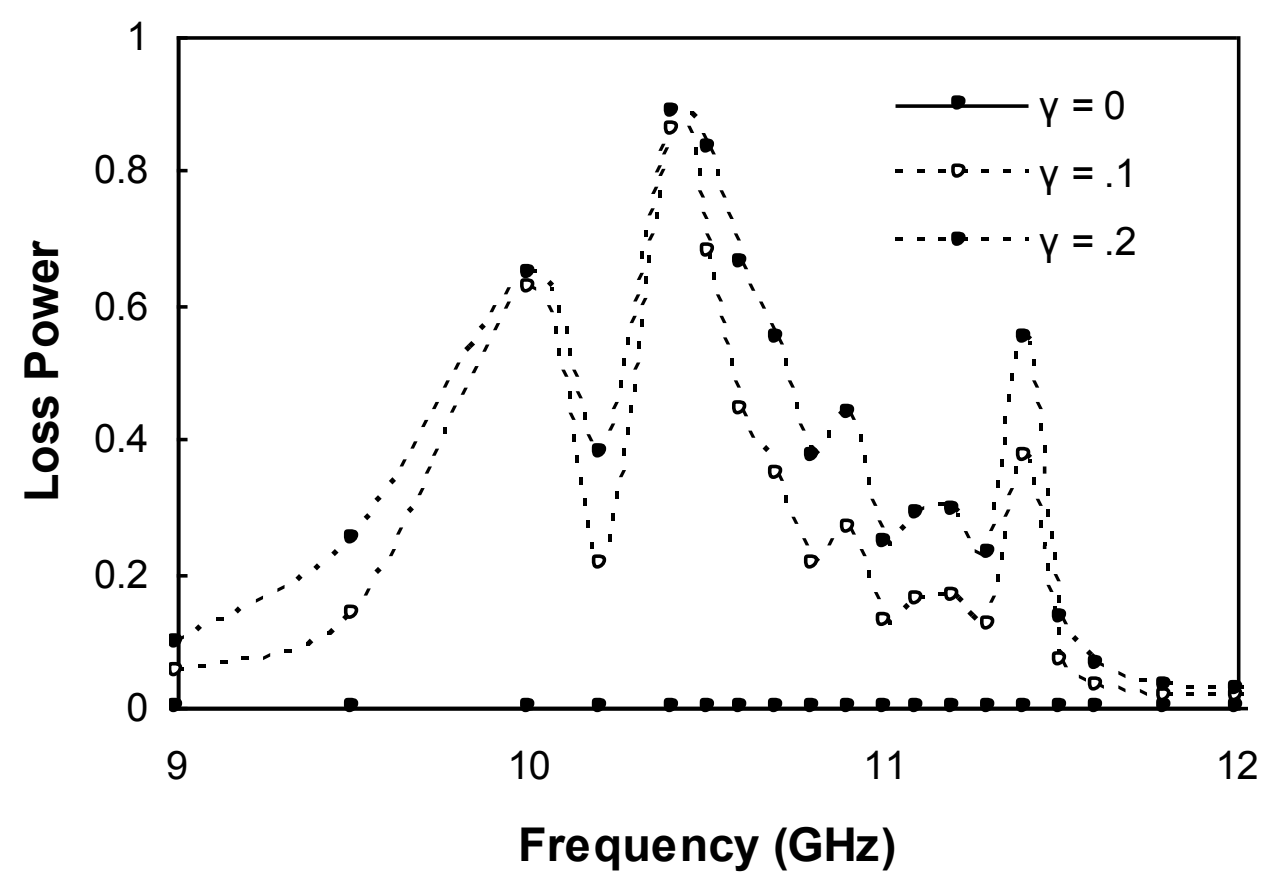

( c )

Fig. 2 (continue). Reflected, transmitted and loss powers as a function of frequency.

Figure 3 illustrates the variation of reflected, transmitted and loss powers with the angle of incidence. The angle of incidence is changed between $0^{\circ}$ and $90^{\circ}$ to realize all possible angles of incidence.

Clearly the reflected power increases while the transmitted and loss powers decrease with the angle of incidence. At $90^{\circ}$ the reflected power is maximum while the transmitted and loss powers are minimum at that angle for any value of dissipation factor.

The role of the dissipation factor is clear at angles below $90^{\circ}$. The reflected and transmitted powers decrease while the loss power increases with the dissipation factor for any angle below $90^{\circ}$.

Figure 4 demonstrates the effect of thickness of LHM on the reflected, transmitted and loss powers respectively at $30^{\circ}$ angle of incidence.

The slab thickness is changed from zero to $30 \mathrm{~mm}$ It is noticed from the figure that the reflected power changes periodically for all dissipation factors.

The transmitted power shows the same property for $\gamma=0 \mathrm{GHz}$, it shows oscillatory decreasing when $\gamma=1$ and $2 \mathrm{GHz}$.

On the other hand the loss power shows oscillatory increasing behavior with the increasing values of the dissipation factor. 


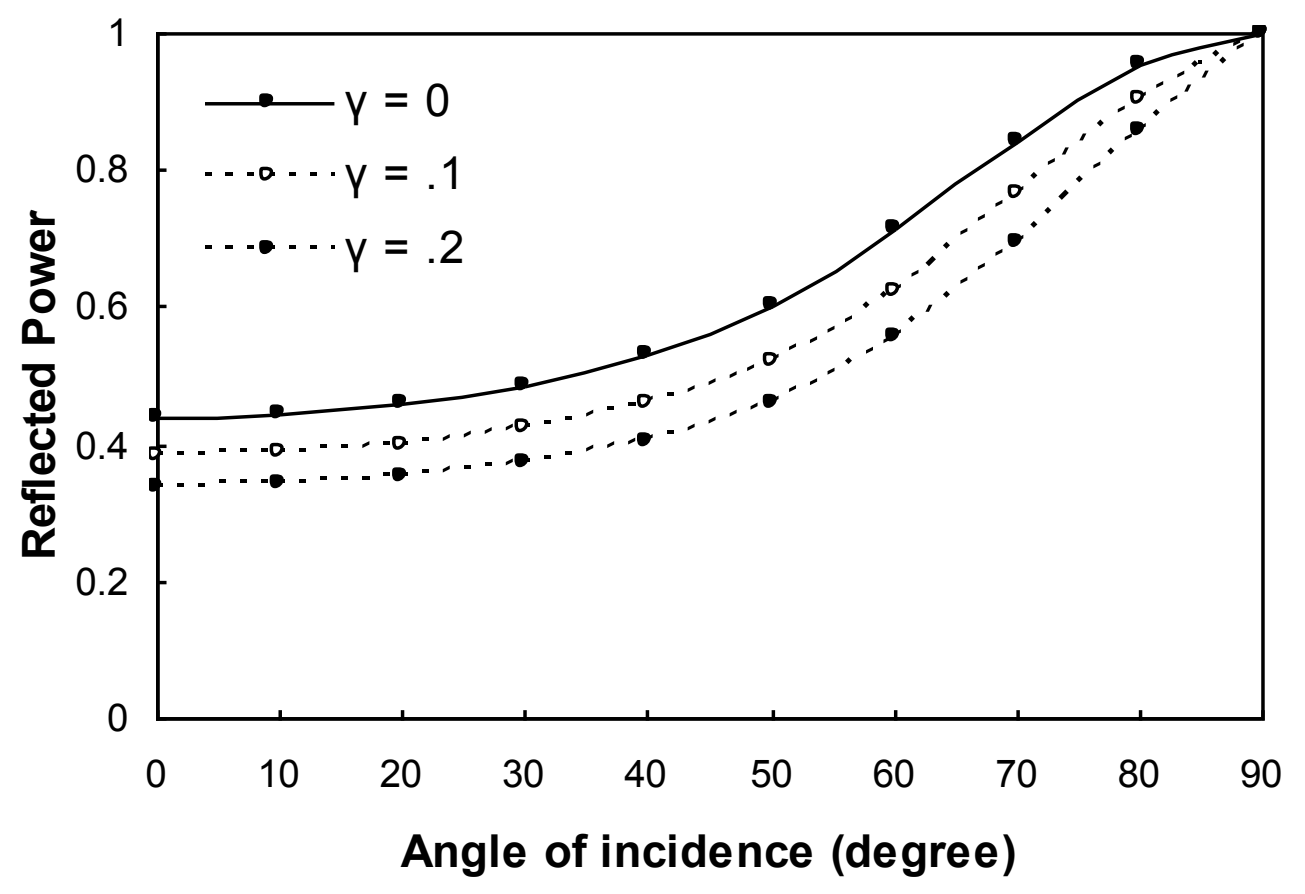

(a)

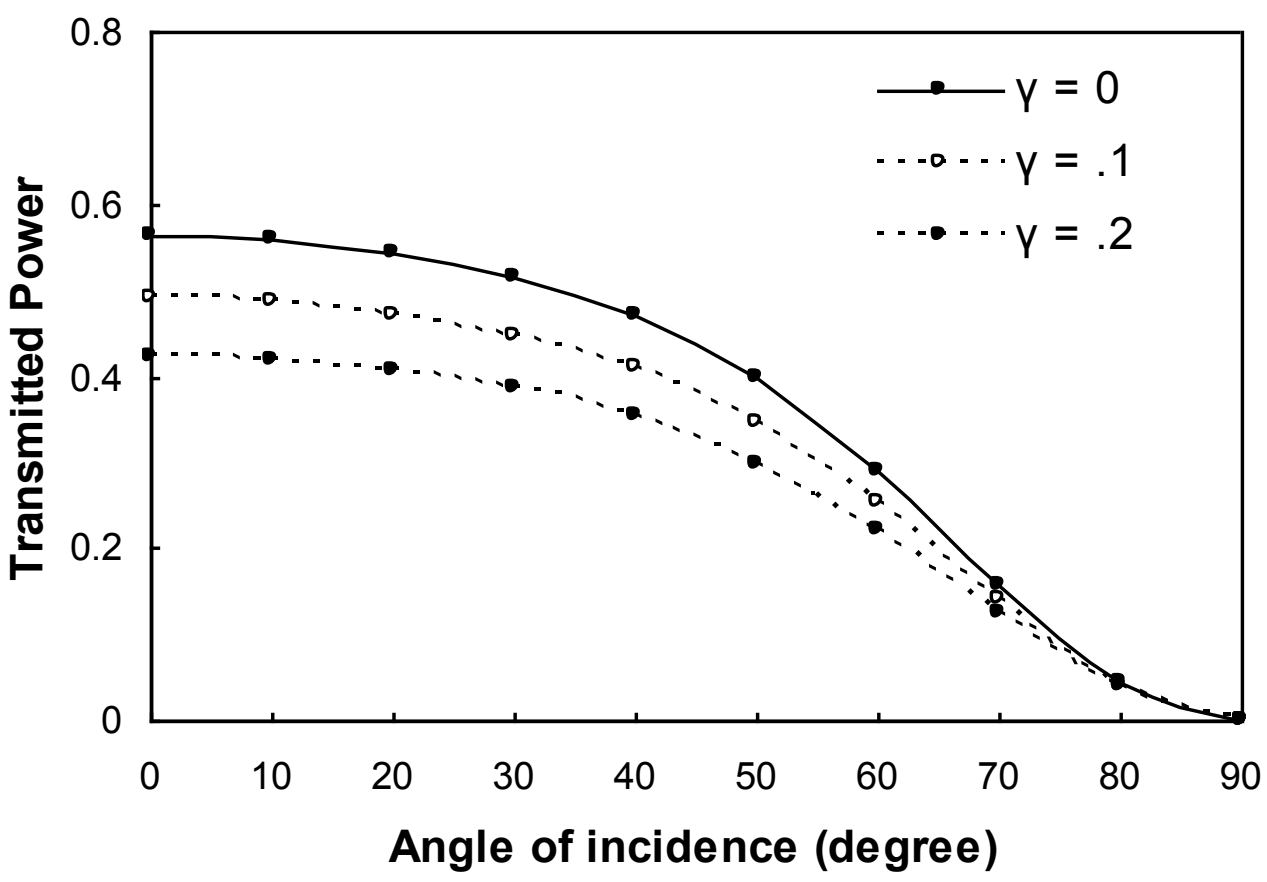

(b)

Fig. 3. Reflected, transmitted and loss powers against the angle of incidence. 


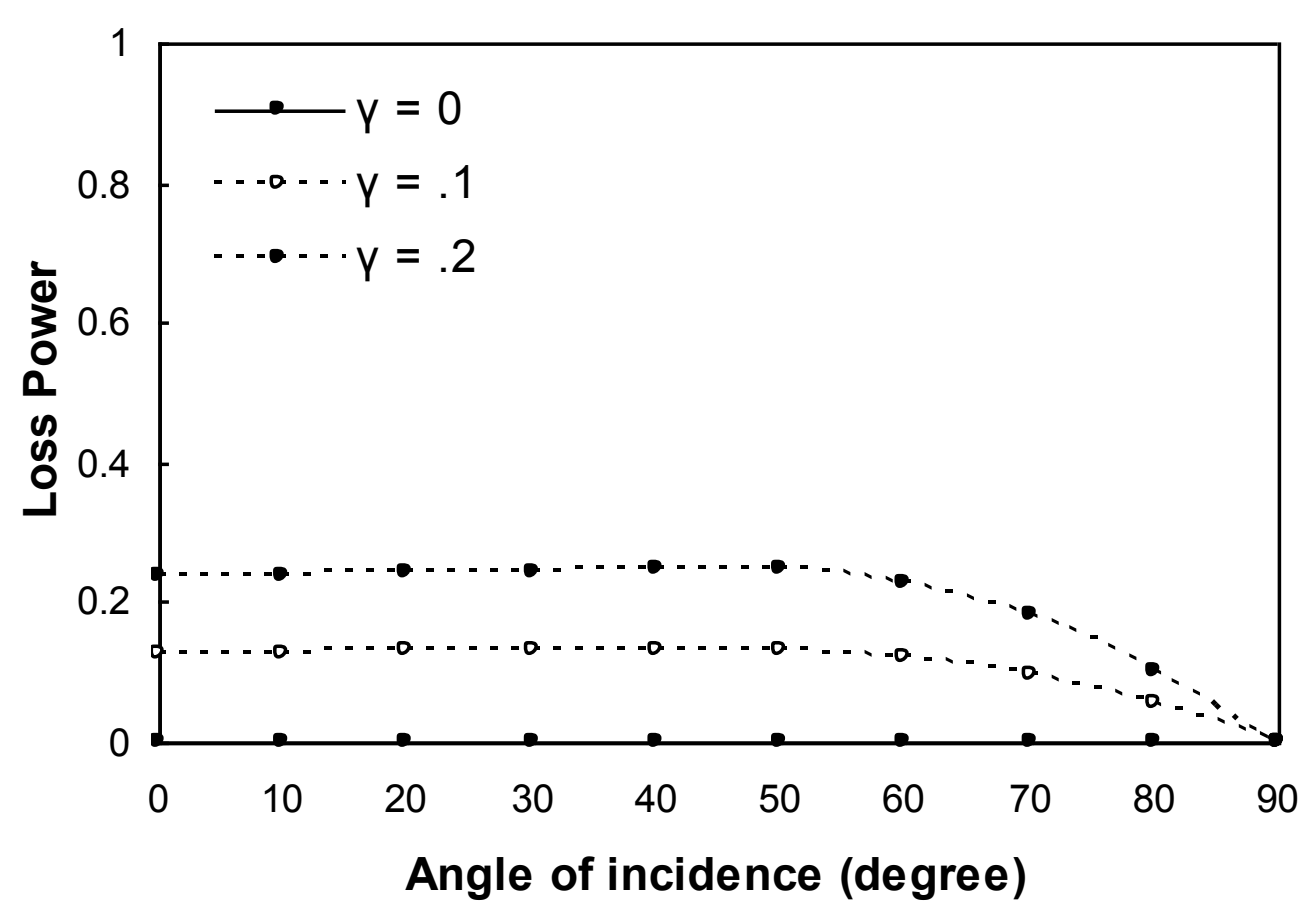

(c)

Fig. 3 (continue). Reflected, transmitted and loss powers against the angle of incidence.

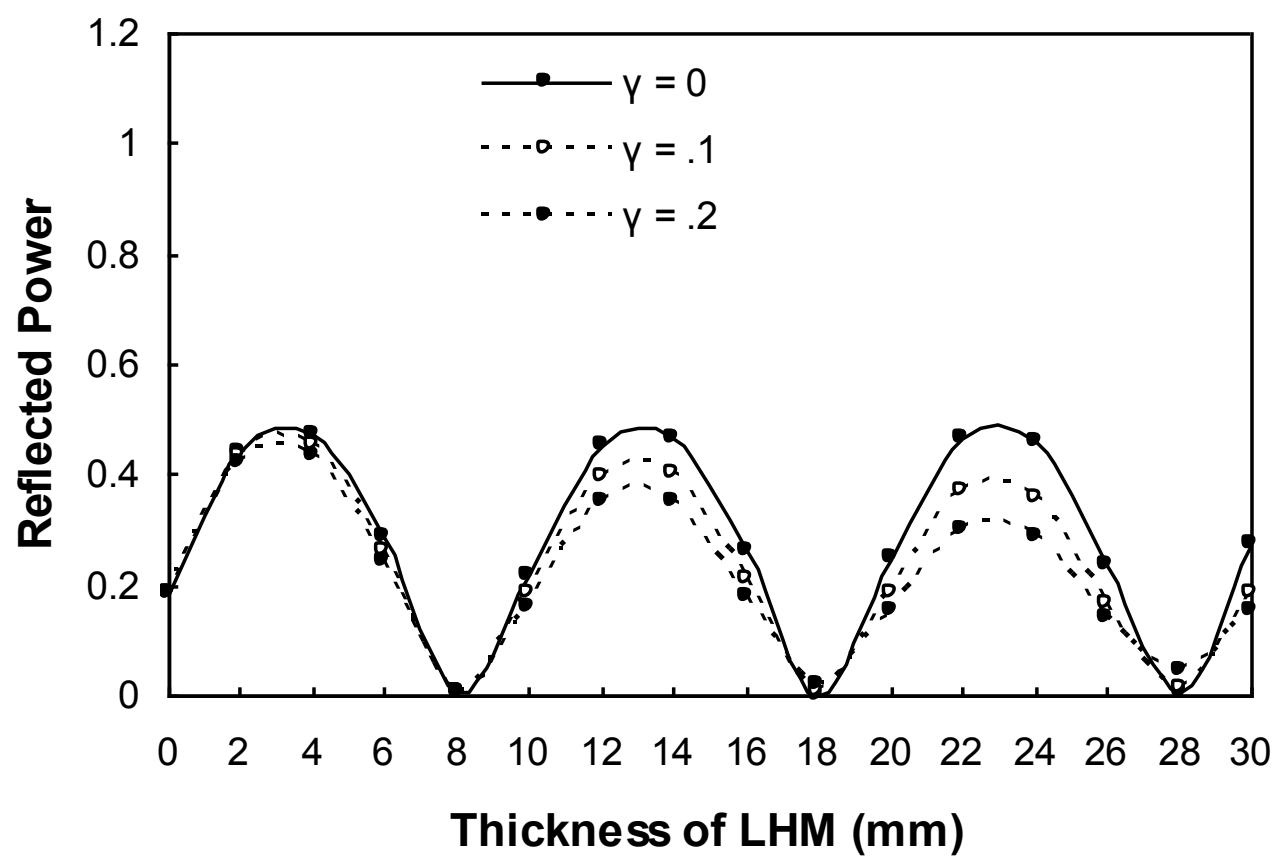

(a)

Fig. 4. Reflected, transmitted and loss powers versus thickness of LHM. 


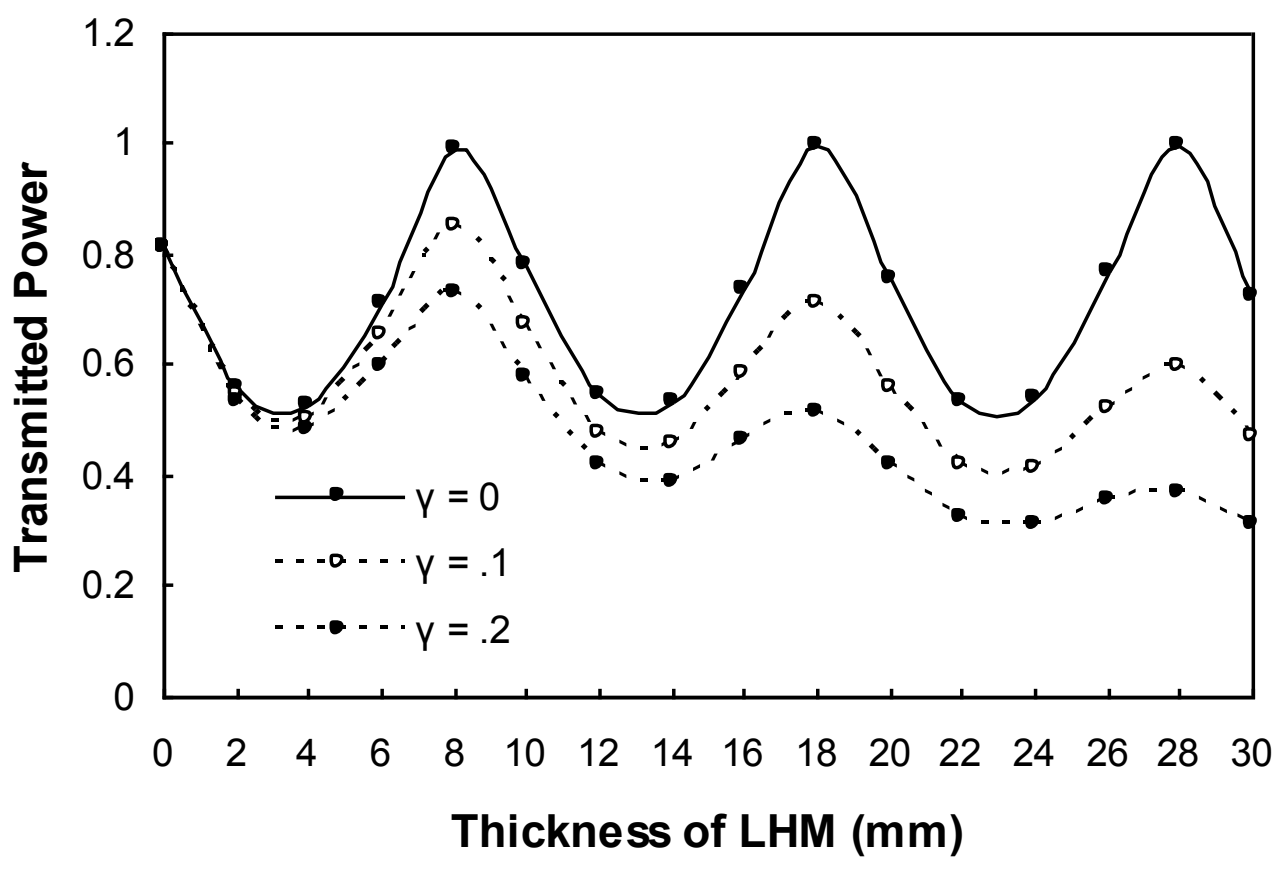

(b)

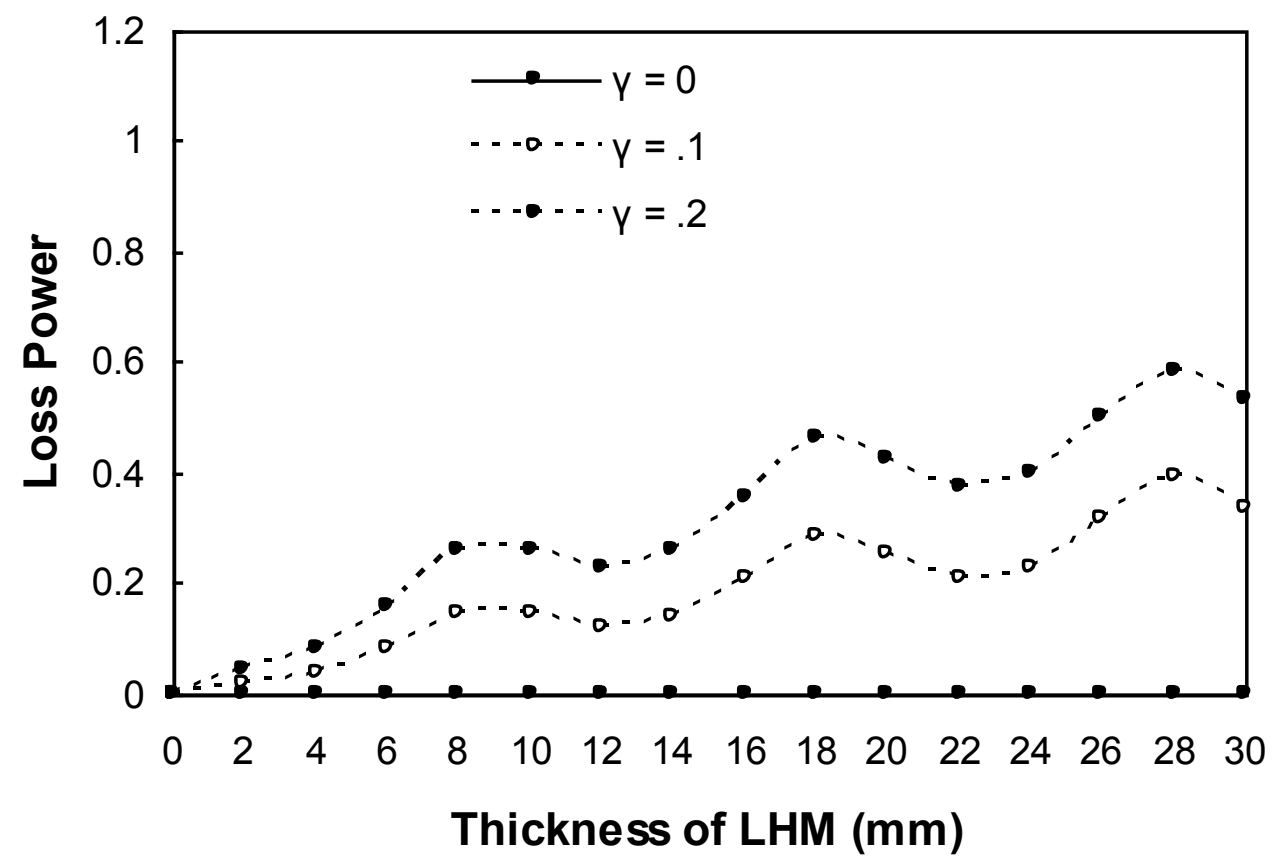

(c)

Fig. 4 (continue). Reflected, transmitted and loss powers versus thickness of LHM. 


\section{CONCLUSION}

The transmission and reflection of electromagnetic waves by a multilayered structure consisting of a pair of LHM and dielectric slabs situated in free space have been studied with effect of the dissipation factor. The followed method is based on Maxwell's equations and matching the boundary conditions for the electric and magnetic fields at each layer interface.

The frequency dependence of $\varepsilon$ and $\mu$ of the LHM is taken into account. The dependence of the reflected, transmitted and loss powers of the considered structure on various parameters have been investigated to observe the effect of the dissipation factor. As can be seen from the numerical results, if the dissipation factor changes, the behaviors of the powers will be affected from this change. Consequently the dissipation factor has an important role in the variations of the powers. The law of conservation of energy given by $[11,14,15]$ is satisfied by our results. The discussed problem is useful for applications which require controlling of reflected and transmitted powers like antenna radome, microwave, millimeter wave and optical devices.

\section{References}

[1] V. G. Veselago, Soviet Phys Uspekhi 10 (1968) 509-514.

[2] J. B. Pendry, A. J. Holden, W. J. Sewart, I. Youngs, Phys. Rev. Lett. 76 (1996) 4773-4776.

[3] J. B. Pendry, A. J. Holden, D. J. Robbins, W. J. Stewart, IEEE Transaction on Microwave Theory and Techniques 47 (1999) 2075-2084.

[4] R. A. Shelby, D. R. Smith, S. Schultz, Science 292 (2001) 77-79.

[5] I. V. Shadrivov, Photonic Nanostruct: Fundam Appl. 2 (2004) 175-180.

[6] N. Garcia, M. Nieto-Vesperinoas, Phys. Rev. Lett. 88 (2002) 7403.

[7] J. A. Kong, Prog. Electromagn. Res. 35 (2002) 1-52.

[8] M. F. Ubeid, M. M. Shabat, M. O. Sid-Ahmed, International Conference for Science and Development (ICSD-IV), 22 - 23 November 2011 The Islamic University of Gaza, Gaza, Palestine, http://iugaza.edu.ps/ar/

[9] I. Kourakis, P. K. Shukla, Phys. Rev. E 72 (2005) 016626.

[10] M. F. Ubeid, M. M. Shabat, M. O. Sid-Ahmed, Natural Science 3 (2011) 328-333.

[11] H. Cory, C. Zach, Microwave and Optical Tech. 40 (2004) 460-465.

[12] C. Sabah, S. Uckun, Piers Online 5 (2009) 601-604.

[13] H. Oraizi, A. Abdolali, IET Microw. Antennas Progpag. 3 (2009) 987-996.

[14] D. D. Stancil, A. Prabhakar, Spin waves, New York: Springer Sience, (2009).

[15] C. Sabah, S. Uckun, Opto-Electron. Rev. 15 (2007) 133-143.

[16] R. A. Shelby, Thesis (PhD.), University of Calefornia, San Diego, Microwave Experiments with Left-Handed Materials, Bell and Howell Information and Learning Company (2001). 